\title{
Principal Properties for Synthetic Screening: Amines
}

\author{
Rolf Carlson, ${ }^{\mathrm{a}, \boldsymbol{}}$ Michal P. Prochazka ${ }^{\mathrm{a}}$ and Torbjörn Lundstedt ${ }^{\mathrm{b}}$
}

aDepartment of Organic Chemistry, University of Umeå, S-901 87 Umeå and ' AB Leo, P.O. Box 839, S-201 80 Malmö, Sweden

\begin{abstract}
Carlson, R., Prochazka, M. P. and Lundstedt, T., 1988. Principal Properties for Synthetic Screening: Amines. - Acta Chem. Scand., Ser. B 42: 157-165.

Principal properties of amines were determined by principal components (PC) analysis of data for a set of 126 amines. Each amine was characterized by seven property descriptors. A two-component PC model was significant according to cross validation, and accounted for $85 \%$ of the variance of the descriptor data.

Primary and secondary amines were described by the same PC model as for the tertiary amines, which indicated that hydrogen bonding is not significantly involved in determining the physical properties of amines.

Factors that determine the chemical behaviour of amines are discussed. Principles for selecting test objects using the principal properties are briefly discussed.
\end{abstract}

Amines are an important class of compounds in organic synthesis. Numerous examples of electrophilic functionalization on the nitrogen can be given. Primary and secondary amines will thus give neutral species in which a proton is displaced. A rough estimate based on the pharmaceutical literature indicates that over $95 \%$ of all commercially available pharmaceuticals are amine derivatives. Tertiary amines are often used as base catalysts and/or proton scavengers.

To explore new synthetic procedures, either for functionalizing amines or using amines as auxiliary reagents, it is desirable that test systems span a wide range of variation. It is also desirable that all important properties of the system are considered in this context.

In as series of papers we have described new principles for the selection of test objects in synthetic explorations. These methods are based on principal components (PC) analysis of property descriptors for series of potential test candidates. The significant components thus obtained are orthogonal measures of systematic variations and are called principal properties. Principal properties have hitherto been reported for organic sol-

*'To whom correspondence should be addressed. vents, ${ }^{1}$ Lewis acids, ${ }^{2}$ aldehydes, ${ }^{3}$ ketones ${ }^{3}$ and amino acids. ${ }^{4}$ We have also discussed how principal properties can be used to explore the entire reaction space. ${ }^{5}$

A study of amine variation in the WillgerodtKindler reaction was based on principal properties of a small set of 29 primary and secondary amines. ${ }^{6}$ However, to be of general and practical interest, selection of test objects must be made from a much larger collection of potential candidates. In this paper, which concludes our series of papers on principal properties for synthetic screening, we report a study of 126 commercially available primary, secondary and tertiary amines.

\section{Data}

The amines were characterized by seven property descriptors. Data are given in Table 1. The descriptors were compiled from different sources. ${ }^{7}$ IR data were also considered initially, but these descriptors were deleted in the final analysis since they were not consistent over the whole data set; e.g. one compilation of literature data on amines $^{7 a}$ contained data for the strongest IR absorption band which, however, referred to different chromophores. IR data were also missing for a number of amines. 


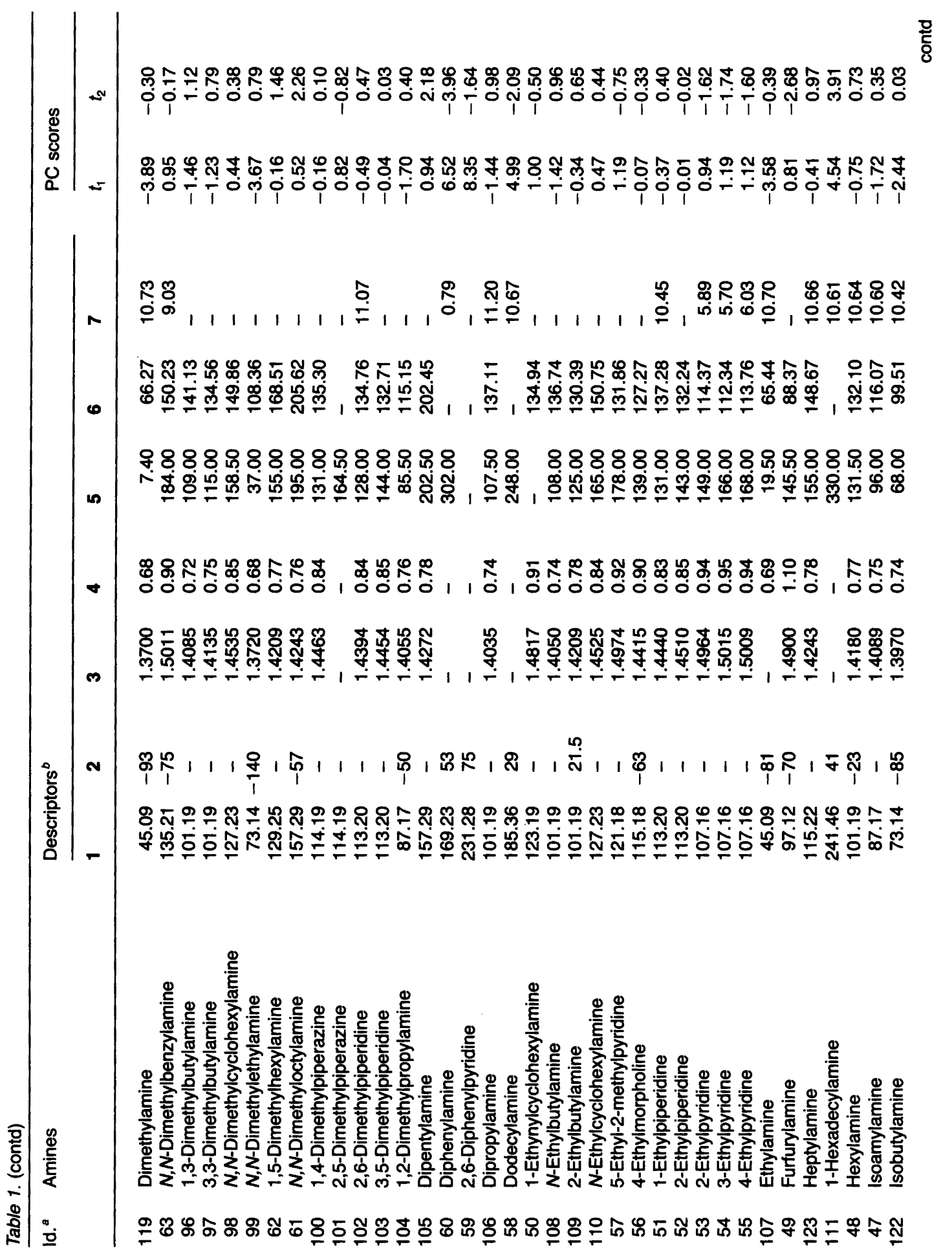


CARLSON ET AL.

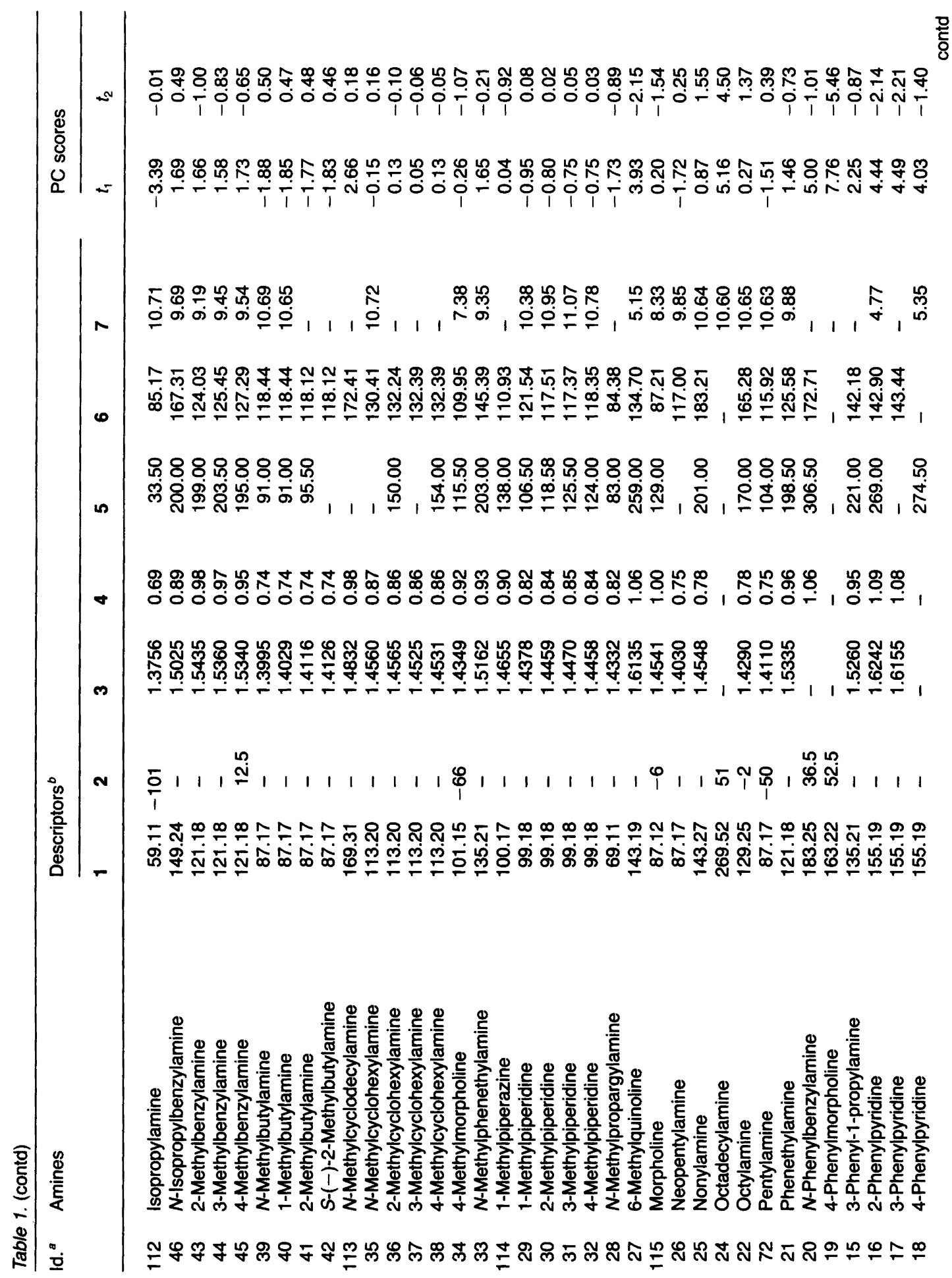




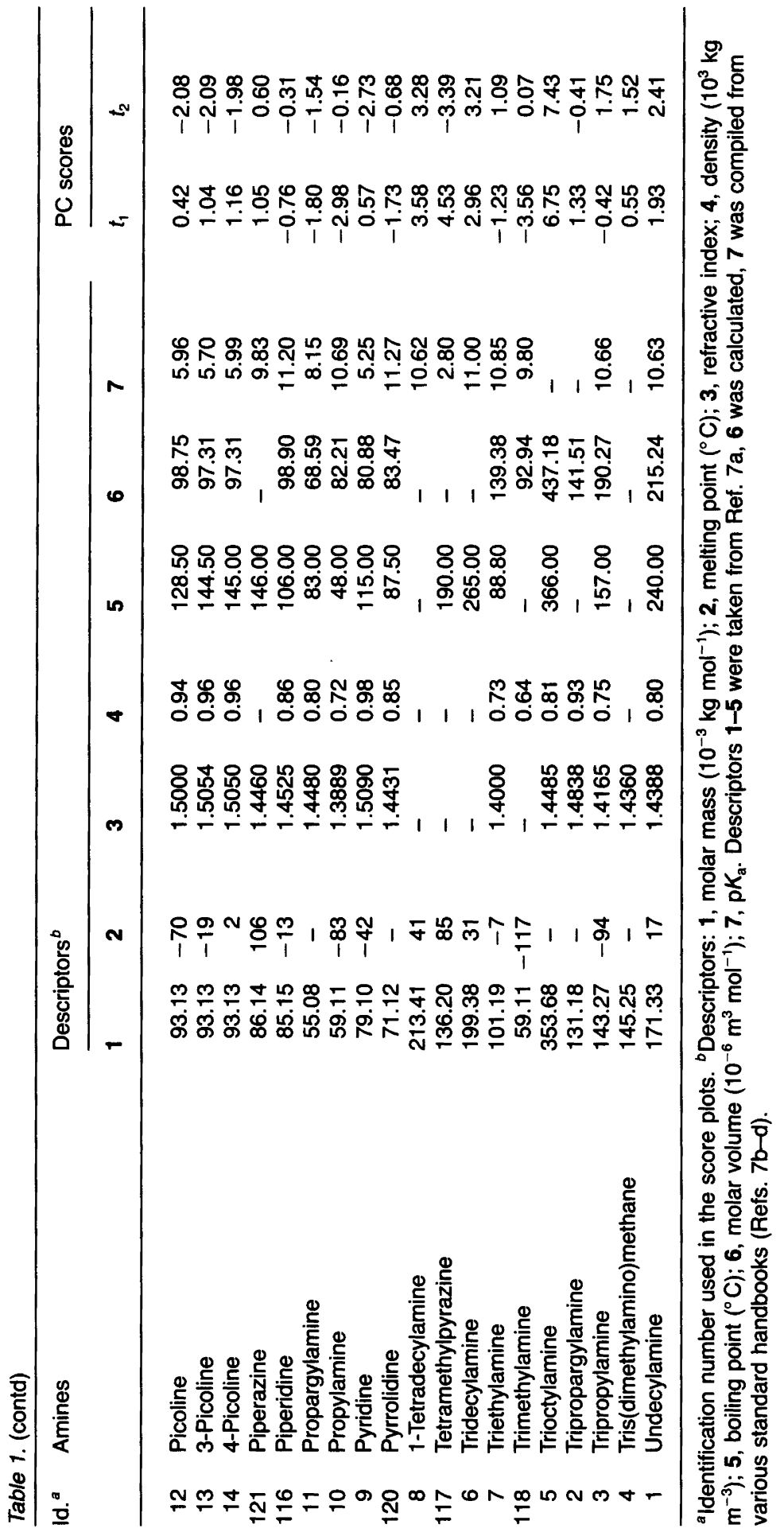




\section{Methods}

The principal properties were determined by fitting PC models to the data in Table 1. The SIMCA program package was used. ${ }^{8}$ The num- ber of significant components was determined by cross validation. ${ }^{9}$ Prior to calculation of the PC models, each descriptor variable was scaled to unit variance (autoscaling). This was done to avoid distorting the variance through the use of

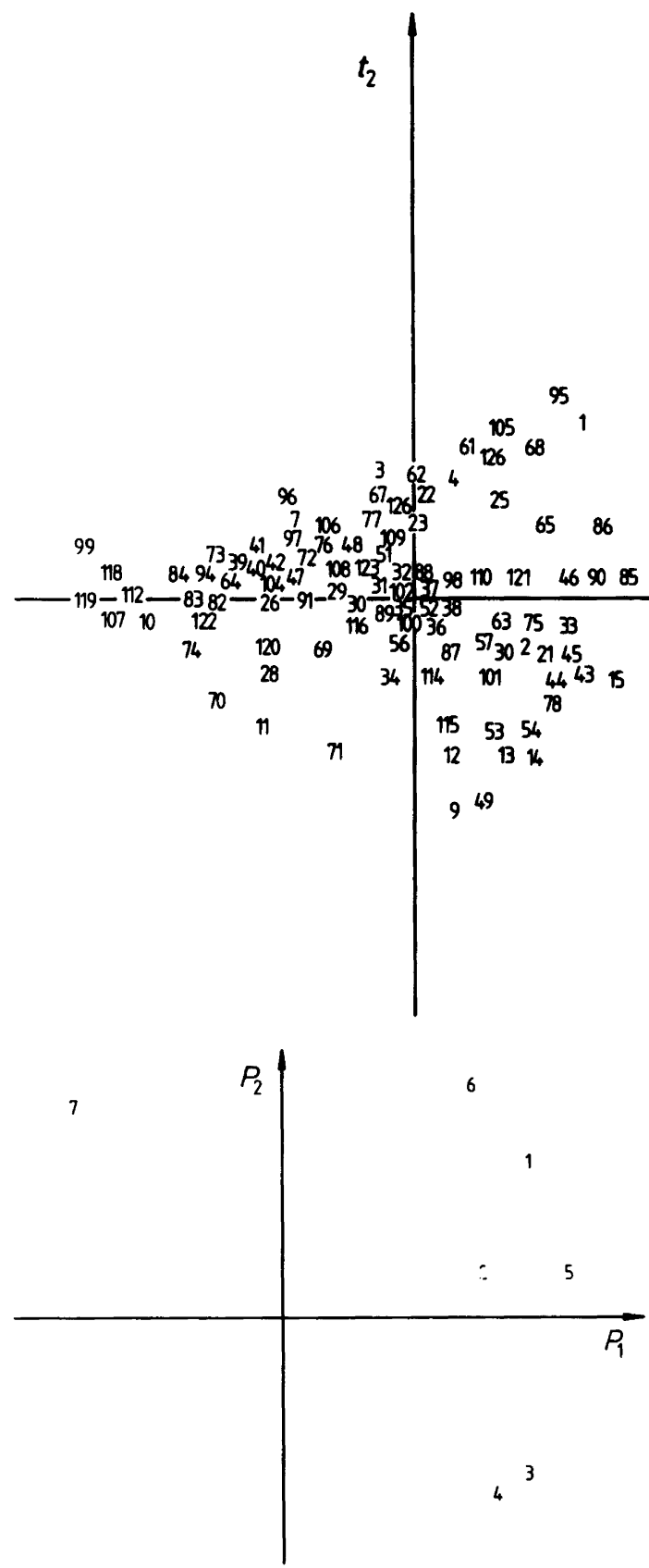

24

m

68

5892

$66 \quad 93$

113

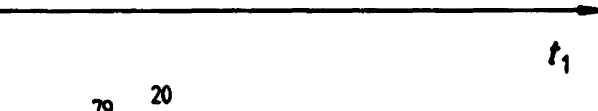

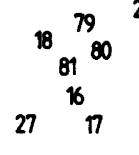

11

60

19
Fig. 1.(a) Score plot of the whole data set in Table 1. (b) Loading plot showing the contribution of the descriptors to the principal properties. 
different units of measurement. Detailed accounts of PC analysis have been given elsewhere. ${ }^{8,10}$

\section{Results}

The data in Table 1 were analyzed as follows: To check the homogeneity of the data, a PC model was calculated from the whole data set. A twocomponent model was significant according to cross validation and accounted for $88 \%$ of the total variance. For some amines, however, several data were missing; to prevent this introducing bias into the model, a calibration set of 95 amines were selected allowing a maximum of two missing data for each amine. A two-component model described $85 \%$ of the variance. The principal properties were then determined by projecting the whole data set down to this model. The PC projections are shown in Fig. 1, and the PC scores ( $t$ values) are listed in Table 1. Two amines, viz. tetramethylpyrazine (No. 117) and piperazine (No. 121) were projected outside the $95 \%$ confidence limits of the model.

Primary and secondary amines can form intermolecular hydrogen bonds which may influence their physical properties. To analyze whether this would require disjoint PC models for tertiary amines and for amines which can give rise to hydrogen bonding, PC models were fitted to training sets of either type of amine. In these training sets, a maximum of two missing data for each amine were allowed. The PC projection for the tertiary amines is shown in Fig. 2. However, projection of the whole data set in Table 1 onto either model afforded almost identical results. This shows that separate models for the two types of amines are not necessary.

\section{Discussion}

Descriptors. Missing data are always a problem when data are compiled for large sets of chemical compounds. This restricts the number of property descriptors, since only easily measured properties are commonly available.

The chemical behaviour of amines depends on at least four factors, viz. basicity, nucleophilicity, solvation and steric environment of the amine nitrogen.

Basicity. The only basicity descriptor generally available for a sufficiently large number of the amines in Table 1 is $\mathrm{p} K_{\mathrm{a}}$ (measured in water). We conclude that this is sufficient for screening purposes since a projection almost identical to that given in Ref. 6 was obtained when the primary and secondary amines included in this previous study were projected onto the model calculated for the amines in Table 1 . The basicity descriptors used to characterize the 29 primary and secondary amines in the previous study were, in addition to $\mathrm{p} K_{\mathrm{a}}$, proton affinity and gas-phase basicity.

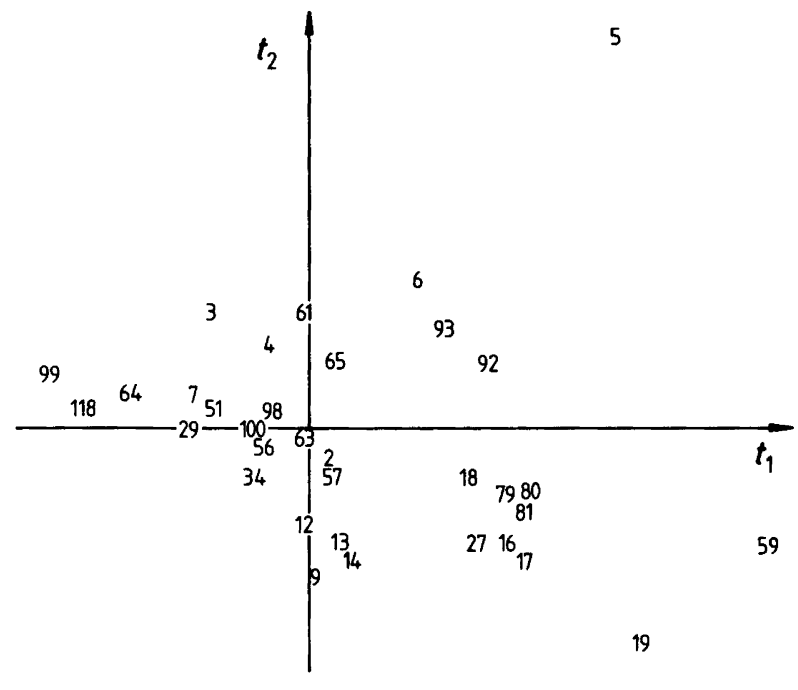

12 Acta Chemica Scandinavica B 42 (1988)
Fig. 2. Score plot for tertiary amines. 
Nucleophilicity. It is difficult to obtain good descriptors for this property. Kinetic data for nucleophilic displacement reactions are available for only a few amines. Since nucleophilicity is, in part, dependent on the properties of the lonepair electrons on the nitrogen, indirect measures related to this property must be used, for instance $\mathrm{p} K_{\mathrm{b}}$ or some other basicity descriptor or refractive index, which is related to polarizability. These descriptors were used in the present study. There are other suitable data e.g. ionization potentials and frontier orbital energies, but these were unfortunately not available for a sufficiently large number of amines.

Solvation. The substrate and reagent may be differently solvated when the solvent is changed, which may alter the reactivity pattern. Principal properties can be used to design screening experiments in which both solvent and reagent are simultaneously varied. ${ }^{5}$

The unexpected result that tertiary amines afford the same PC model as primary and secondary amines shows that hydrogen bonding does not significantly influence physical properties such as melting point, boiling point and density. These properties must therefore depend on weak dispersion forces. Such forces are responsible for solvation in aprotic non-polar organic solvents commonly used in synthetic reactions. It has previously been shown that in absence of strong interactions, several properties in the liquid state can be adequately described by PC models. ${ }^{11}$

In strongly associated protic solvents, hydrogen bonding will be involved in solvation. This property is not covered by the descriptors used in the present study. In a situation in which both the amine and the solvent are to be jointly considered, such interaction will be accounted for by the principal properties of the solvent.

Steric environment of the amine nitrogen. This factor is not treated in the present analysis. It is suggested that steric factors should be considered separately as an additional criterion for selection based on the principal properties. A discussion of a similar problem, viz. selection of aldehydes and ketones on the basis of their principal properties, is given in Ref. 3.

Selection of test compounds. PC analysis reveals the systematic variation in all the descriptors.
The score plot portrays this variation. Hence, such projections can be used to achieve selection of test objects in which all properties are considered simultaneously. Thorough discussions of the principles of such selections have been given in previous papers. ${ }^{1-6}$ Here, only a brief summary is presented:

(A) Select test objects that are projected on the periphery of the score plot. This will give a selection which spans a large range in all properties considered.

(B) Select test objects that are uniformly spread over the score plot. This will give a selection in which a uniform spread in all properties is achieved.

\section{Conclusions}

The principal properties of amines are described by two principal components. The PC model accounted for $85 \%$ of the variation in a set of seven property descriptors. The property descriptors are related to factors which govern the chemical reactivity. Hence, an experimental design based on the principal properties will allow for selection of test compounds so that a sufficient variation in all important properties is achieved. This may lead to an increased efficiency in experimentation by allowing test objects to be easily selected so that a maximum of information can be obtained in the individual experiments.

\section{Calculations}

The calculations were carried out on a IBM PC-XT or IBM-compatible microcomputers. The SIMCA program package (SIMCA 3-B version) was used for modelling. The program is available from Sepanova $A B$, Östrandsvägen 14, S-112 43 Enskede, Sweden or from Principal Data Components, Shepard Blvd., Columbia, Missouri 65201, USA.

Acknowledgements. Financial support from the Swedish Natural Science Research Council and from the National Swedish Board for Technical Development is gratefully acknowledged. The authors thank Mr. Stephen Swanson for linguistic revision of the manuscript. 


\section{References}

1. Carlson, R., Lundstedt, T. and Albano, C. Acta Chem. Scand., Ser. B 39 (1985) 79.

2. Carlson, R., Lundstedt, T., Nordahl, Å. and Prochazka, P. Acta Chem. Scand., Ser. B 40 (1986) 522.

3. Carlson, R., Prochazka, M. and Lundstedt, T. Acta Chem. Scand., Ser. B 42 (1988) 145.

4. Hellberg, S., Sjöström, M., Skagerberg, B. and Wold, S. J. Med. Chem. 30 (1987) 1126.

5. (a) Carlson, R. and Lundstedt, T. Acta Chem. Scand., Ser. B 41 (1987) 164; (b) Carlson, R. Chem. Scr. 27 (1987) 545.

6. Lundstedt, T., Carlson, R. and Shabana, R. Acta Chem. Scand., Ser. B 41 (1987) 157.

7. (a) The Aldrich Microfiche Library of Chemical Indices, Aldrich Chemical Company Inc., 1987; (b) Vogel, A. Textbook of Practical Organic Chemistry, 4th ed., Longmans, London 1978; (c) Handbook of Chemistry and Physics, 57th ed., CRC
Press Inc., Boca Raton, FL, USA 1976; (d) The Merck Index, 9th ed., Merck and Co Inc., Rathway, N. J., USA 1976.

8. (a) Wold, S. Pattern Recognition 8 (1976) 127; (b) Sjöström, M. and Wold, S. In: Kowalski, B. R., Ed. Chemometrics, Theory and Applications, American Chemical Society Symposium Series 52, American Chemical Society, Washington D.C. 1977.

9. Wold, S. Technometrics 20 (1978) 397.

10. (a) Joliffe, I. T. Principal Components Analysis, Springer, New York 1986; (b) Wold, S., Albano, C., Dunn, W. J., III, Edlund, U., Esbensen, K., Geladi, P., Hellberg, S., Johansson, E., Lindberg, W. and Sjöström, M. In: Kowalski, B. R., Ed., Chemometrics, Mathematics and Statistics in Chemistry, D. Riedel Publ. Co., Dordrecht, Holland 1984.

11. Cramer, R. D., III. J. Am. Chem. Soc. 102 (1980) 1849.

Received September 14, 1987. 